\title{
A very rare cause of protein losing enteropathy: Gaucher disease
}

\author{
Mehmet Akif Göktaş ${ }^{1 \oplus}$, Ersin Gümüşs ${ }^{1 \oplus}$, Hülya Demir ${ }^{1 \oplus}$, \\ Hayriye Hızarcıoğlu Gülşen ${ }^{1 \odot}$, İnci Nur Saltık Temizel ${ }^{1 \oplus}$, Hasan Özen ${ }^{1 \oplus}$, \\ Şafak Güçer ${ }^{2 \odot}$, Aysel Yüce ${ }^{1 \odot}$ \\ ${ }^{1}$ Division of Pediatric Gastroenterology, Hepatology and Nutrition, ${ }^{2}$ Pediatric Pathology Unit, Department of Pediatrics, Hacettepe \\ University Faculty of Medicine, Ankara, Turkey.
}

\begin{abstract}
Background. Mesenteric lymphadenopathy is a rare manifestation of Gaucher disease (GD) in children and can be accompanied by protein losing enteropathy (PLE). PLE is a difficult-to-treat complication of GD. To date, only a few pediatric GD cases with PLE and massive mesenteric lymphadenopathies have been reported.

Case. Here, we report a girl with chronic neuronopathic GD, whose disease course was complicated by massive mesenteric lymphadenopathies with resultant protein losing enteropathy despite a regular and appropriate enzyme replacement therapy of $60 \mathrm{IU} / \mathrm{kg} / \mathrm{biweekly}$ until the development of mesenteric lymphadenopathies and $120 \mathrm{IU} / \mathrm{kg} /$ biweekly thereafter.

Conclusions. PLE is a devastating and life threatening complication of GD developing despite long term use of high dose ERT. Clinicians should be alert for this complication particularly in GD patients presenting with progressive abdominal distension, edema, ascites and diarrhea or in patients who have already developed mesenteric lymphadenopathies. Timely diagnosis may allow early intervention with previously suggested surgical or medical treatment options. Although there is no specific and effective treatment, surgical and aggressive medical interventions in addition to ERT were reported to relieve diarrhea and halt progression of mesenteric lymphadenopathies.
\end{abstract}

Key words: enzyme replacement therapy; lymphadenopathy; lysosomal storage disorder; Gaucher disease.

Gaucher disease (GD) is a rare, autosomal recessive genetic disorder caused by deficiency of lysosomal enzyme, glucocerebrosidase, which results in the accumulation of glucocerebrosideladen macrophages (Gaucher cells) in the reticuloendothelial system. $^{1}$ Mesenteric lymphadenopathy is a rare manifestation of GD in children and can be accompanied

Ersin Gümüş

ersin.gumus@hacettepe.edu.tr

Received 13th October 2020, revised 18th November 2020, accepted 20th November 2020.

This study was presented at the 16th Annual World Symposium held between February 10-13, 2020 in Orlando (FL, USA) as a poster presentation and published as an abstract in Molecular Genetics and Metabolism (doi:10.1016/j.ymgme.2019.11.149). by protein losing enteropathy (PLE). PLE is a difficult-to-treat complication of GD. To date, only a few pediatric GD cases with PLE and massive mesenteric lymphadenopathies have been reported. ${ }^{1-4}$ Here, we report a girl with chronic neuronopathic GD whose disease course was complicated by massive mesenteric lymphadenopathies with resultant protein losing enteropathy despite a regular and appropriate enzyme replacement therapy (ERT).

\section{Case Report}

The patient was the first child of nonconsanguineous parents and the diagnosis of GD was made at the age of 18 months. At the time of diagnosis, she had severe hepatosplenomegaly 
and pancytopenia (Table I). Her neurological examination was completely normal initially. Gaucher cells were seen on bone marrow aspirate and leukocyte enzyme assay showed decreased beta glucocerebrosidase activity. Diagnosis was confirmed with genetic testing showing a compound heterozygosity (L393V and L444P mutations) in the GBA gene. ERT with imiglucerase at an intravenous dose of $60 \mathrm{IU} / \mathrm{kg} /$ biweekly was started immediately. A splenectomy was performed because of hypersplenism and intractable pancytopenia at the age of 3.5 years (Table I). On routine follow-up visit at the age of 5 years, physical examination revealed an ill-defined abdominal mass. Ultrasound and computed tomography (CT) of abdomen demonstrated a lobulated, heterogeneous mass reaching a diameter of 8 $\mathrm{cm}$ and consisting of multiple conglomerated mesenteric lymphadenopathies with cystic degeneration and calcification (Fig. 1). A simultaneously performed CT of the chest also revealed accompanying axillary, supraclavicular, paratracheal, and hilar lymphadenopathies. A tru-cut biopsy from abdominal mass was performed with a high suspicion of lymphoma. Histopathology of biopsy specimens revealed a completely effaced lymph node structure by diffuse infiltration of Gaucher cells with no evidence of malignancy. ${ }^{5}$ Treatment dose of imiglucerase was escalated to $120 \mathrm{IU} / \mathrm{kg} / \mathrm{biweekly}$. The patient remained clinically stable without any change in the size of lymph nodes for 7 years following dose escalation. Imiglucerase-specific antibodies were not detected at routine controls. At 10 years of age oculomotor apraxia and hearing loss developed and she was diagnosed as type 3 Gaucher disease.

The patient was hospitalized with the symptoms of chronic watery diarrhea and progressive abdominal distention at the age of 12 years. Physical examination showed ascites and generalized edema in addition to a palpable abdominal mass. Laboratory examinations revealed hypoalbuminemia and hypoproteinemia with electrolyte imbalance and alkalosis. Hepatic synthetic function tests and urine evaluation for albuminuria were normal. Acute phase reactants were negative. There was no evidence of pathogenic bacteria, viruses and parasites in stool examinations. Echocardiographic evaluation was normal. Fecal alpha-1 antitrypsin level was elevated suggesting an excessive protein loss from the intestinal system. Abdominal ultrasound confirmed the presence of moderate ascites and persisting giant conglomerated mesenteric lymphadenopathies (Table I). Gastrointestinal symptoms were relieved partially with supportive treatment including intermittent albumin infusions, fluid and electrolyte replacement, medium chain triglyceride (MCT)enriched oil supplementation and parenteral
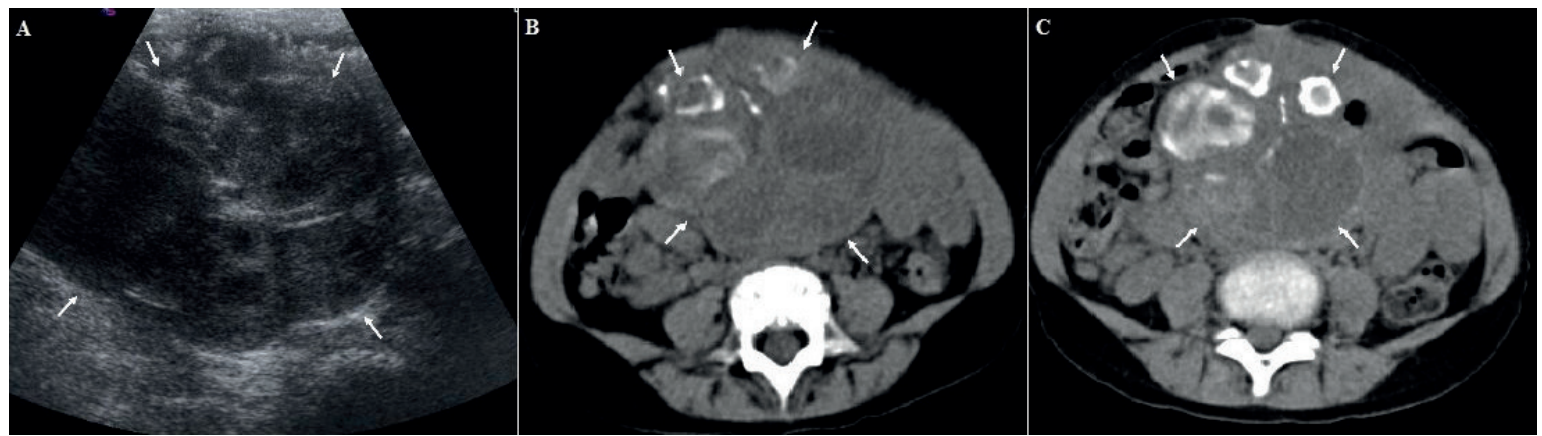

Fig. 1. Ultrasound imaging (A) and CT scan of the abdomen (B) showing a large, lobulated and heterogeneous abdominal mass consisting of conglomerated mesenteric lymphadenopathies some of which exhibiting cystic degeneration and calcification (5 years of age). Follow-up CT scan of the abdomen (C) showing similar radiological findings with areas of cystic breakdown and more extensive calcification in enlarged lymph nodes $(7$ years of age). No regression in radiological findings was observed despite high dose ERT (120 IU/kg/biweekly). 


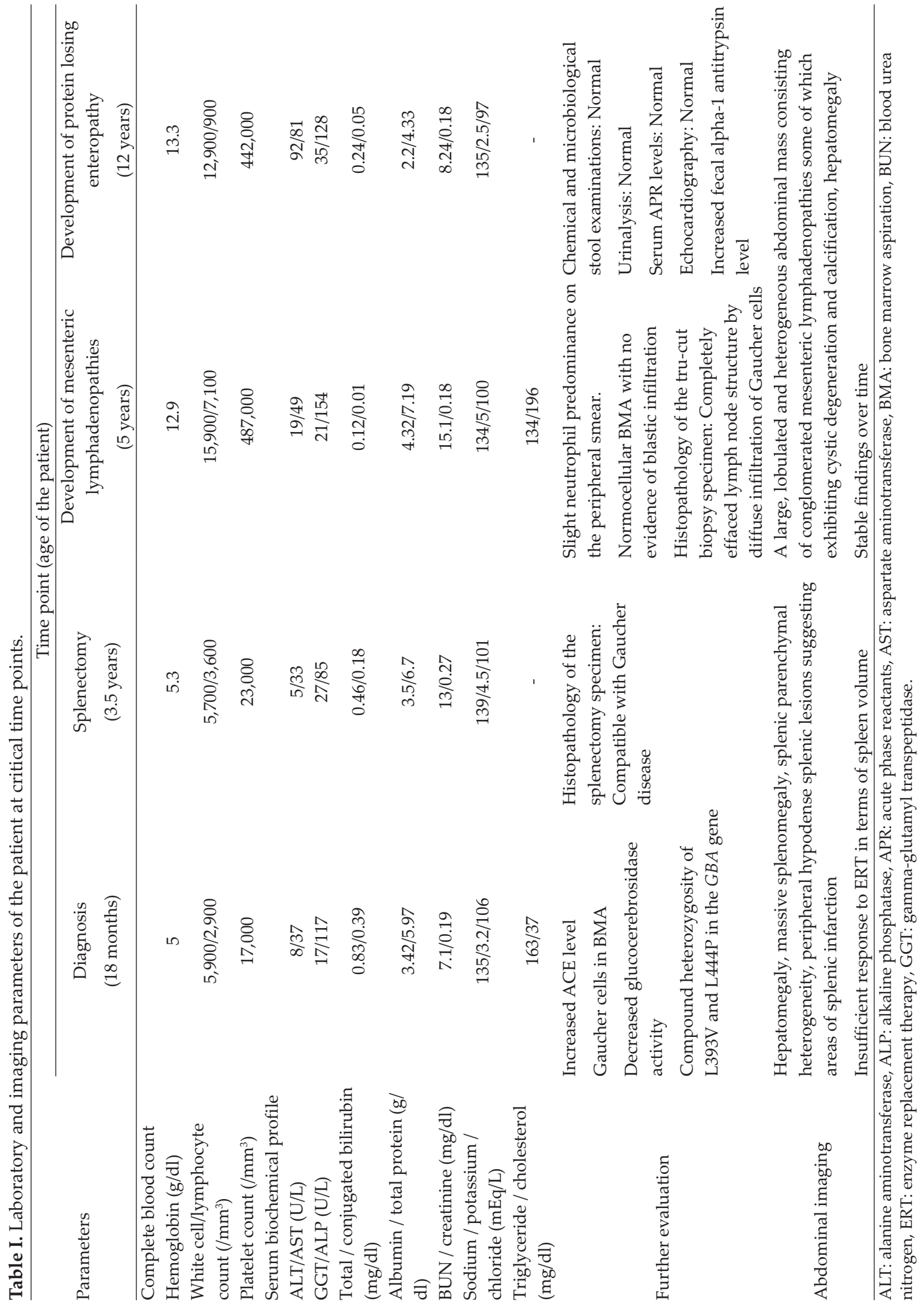


nutrition while continuing high dose ERT. However, the patient died of complications of PLE during outpatient follow-up.

Informed consent was received from the family.

\section{Discussion}

Including the present case, only five pediatric cases with GD whose disease course were complicated with PLE have been reported to date (Table II) ${ }^{1-4}$ Parallel with our observations, all previously reported cases were on ERT before presenting with this life threatening complication. ERT is a safe and effective option for the treatment of visceral and hematological manifestations of GD; however, central nervous system, lymph nodes and lungs have been reported to show insufficient response to this treatment or even unresponsive to ERT compared to other tissues. ${ }^{6}$ It is postulated that these organs are relatively inaccessible to intravenously administered enzyme. The mechanistic basis for poor permeability of enzyme to lungs and lymph nodes is still a matter of debate. Effacement of normal lymph node architecture with Gaucher cells and resultant histological changes such as fibrosis and calcification may reduce drug access to lymph nodes.

Calcified mesenteric lymphadenopathy seems to be a common finding in GD complicated by PLE. Autopsy of a boy with GD who developed progressive mesenteric lymphadenopathies and PLE showed an acellular material with areas of calcification in place of normal lymphoid tissue. ${ }^{7}$ Lymph node calcification was also reported previously in a 16-year-old boy and in a 4.6-year-old boy both of whom had neuronopathic GD and PLE. ${ }^{2,3}$ We also observed progressive calcification of mesenteric lymph nodes in our patient (Fig. 1).

Another contributing factor might be the development of antibodies against the enzyme. Neutralizing antibodies might possibly lead to a reduction in treatment efficacy. Lee et al. ${ }^{2}$ reported a patient who was tested positive for antibodies to enzyme and developed progressive mesenteric lymphadenopathy with resultant PLE.

Splenectomy might also be considered as a contributing factor in the development of lymphadenopathies as it may lead to deposition of substrates in other reticulaendothelial organs. ${ }^{8}$ It is recommended to spare splenectomy for cases of splenic rupture or cases not responding to well conducted ERT with persistent severe cytopenia related to massive splenomegaly, as was the case in the present patient. To our knowledge none of the previously reported patients had a history of splenectomy. Removal of the spleen might have contributed to the enlarged mesenteric lymph nodes in our patient and played an indirect role in the development of PLE.

Lymph node involvement in GD is not restricted to intraabdominal region. Abdelwahab et al. ${ }^{4}$ reported eight children with GD type 1 and 3 developing mediastinal lymphadenopathy in addition to mesenteric lymph node enlargement and underscored the importance of awareness of this complication as it can easily be confused with malignancy both clinically and radiologically. Despite being less prominent, mediastinal, axillary and supraclavicular lymphadenopathies were also present in our patient and we had to perform a biopsy to rule out lymphoma. ${ }^{5}$

All patients in the literature including the present case were chronic neuronopathic GD patients carrying L444P mutation in GBA gene (Table II). However, it is impossible to make a definitive conclusion regarding phenotypegenotype relationship as there is very limited data. Although PLE has not been reported with non-neuronopathic phenotype, mesenteric lymphadenopathies were also reported to develop in children with type 1 GD. ${ }^{4}$

PLE in a GD patient most likely results from secondary lymphatic obstruction. Enlarged mesenteric lymph nodes block mesenteric lymphatic outflow resulting in 


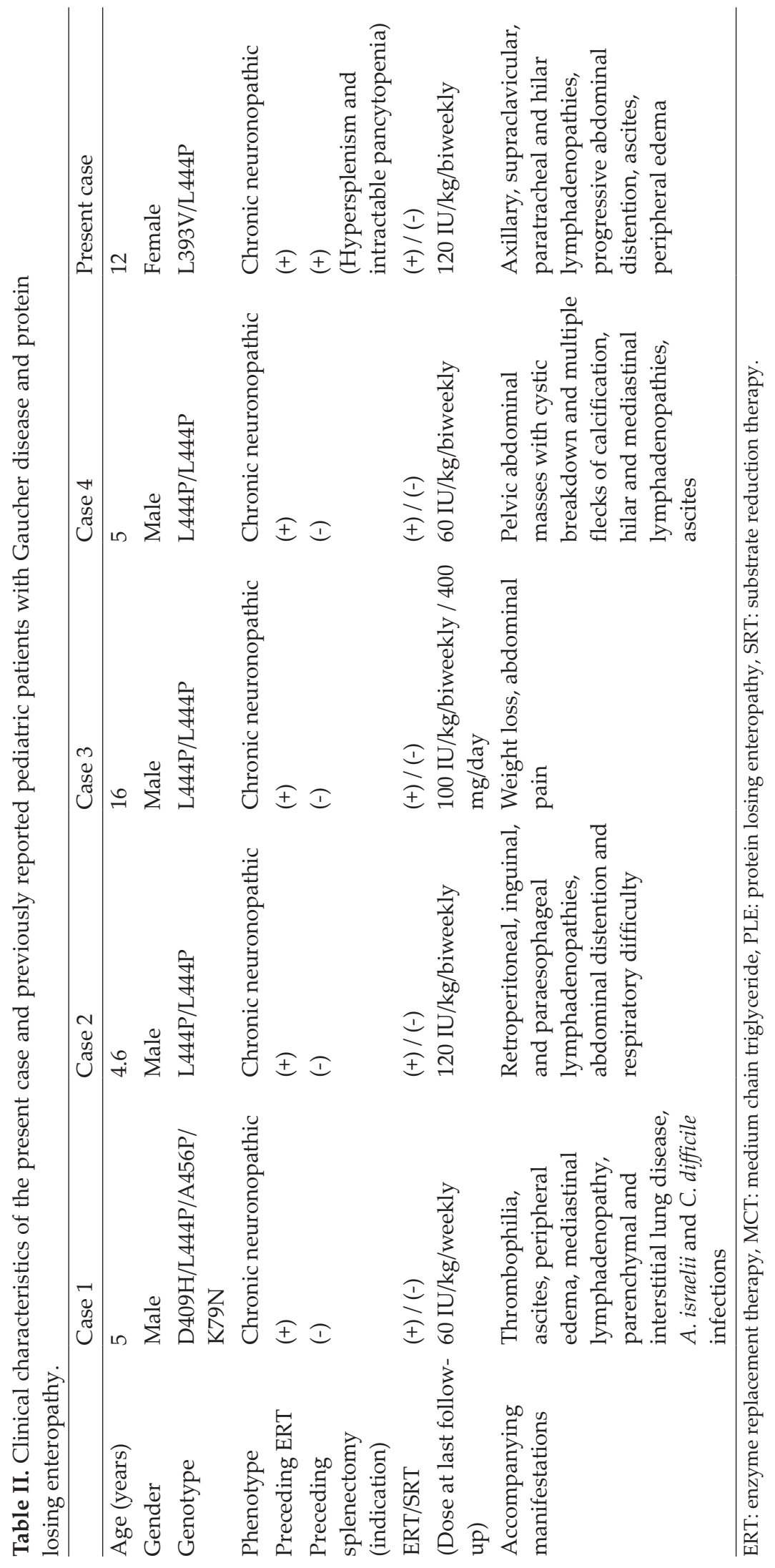




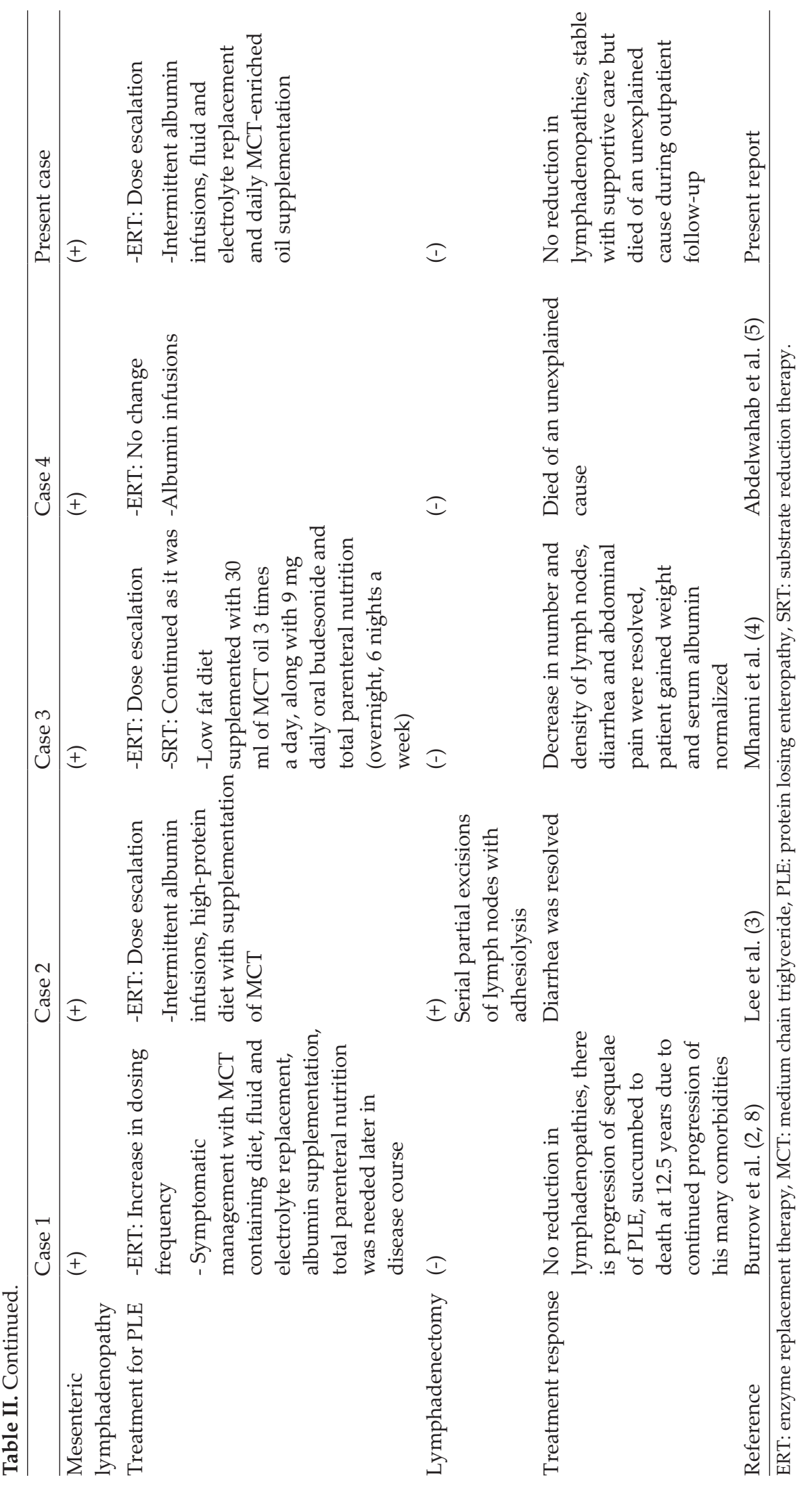


increased lymphatic pressure and intestinal lymphangiectasia which eventually leads to PLE. Development of PLE seems to deteriorate disease course by causing further complications. Difficult-to-control thrombophilia with multiple venous thromboses, need for recurrent hospitalizations due to the severe diarrhea complicating with abdominal distention and respiratory difficulty, and growth retardation with significant weight loss were reported previously in these patients. ${ }^{1-3}$ Our patient was clinically stable until development of PLE even in the time period between detection of giant lymph nodes and initiation of PLE associated diarrhea. After that, she was hospitalized several times for supportive treatment before she succumbed to death.

There is no specific and effective treatment for mesenteric lymphadenopathies and PLE in GD. Neither increasing intensity of ERT nor switching to a combination therapy with substrate reduction therapy seems to be effective. ${ }^{3}$ Surgical excision of lymph nodes was recommended by Lee et al. ${ }^{2}$ They reported that diarrhea was resolved with serial partial excision of lymph nodes with adhesiolysis and concluded that surgical intervention might help control the serious complication of PLE. ${ }^{2}$ However, it should be considered if removal of enlarged lymph nodes will result in deposition of Gaucher cells in other organs as it does in splenectomy.

Another successful treatment approach was reported by Mhanni et $\mathrm{al}^{3}$ In addition to increasing ERT dose in combination therapy, they provided a low fat diet supplemented with MCT oil, along with oral budesonide and total parenteral nutrition. They achieved clinical, laboratory and even radiological improvement with this treatment regimen. ${ }^{3}$ Although we followed a similar management approach except corticosteroid use, our patient failed to respond to supportive treatment and showed an intractable disease course. The patient Mhanni et al. reported might have benefited from budesonide therapy. Budesonide has been used in PLE developing after Fontan procedure as a common and devastating complication. A recent meta-analysis showed that it is effective in ameliorating serum albumin levels in this setting. ${ }^{9}$

Long-term complications of GD which were not observed before the availability of ERT are becoming more apparent with increased life expectancy due to ERT. All five reported GD cases with PLE belong to post-ERT era. Mesenteric lymphadenopathy is not always accompanied by PLE highlighting nonpredictability of the development of this complication. As we still do not know in whom and when these complications will develop, clinicians should be alert to this life threatening complication particularly in GD patients presenting with progressive abdominal distension, edema, ascites, and diarrhea or in patients who have already developed mesenteric lymphadenopathies. Timely diagnosis may allow early intervention with previously suggested surgical or medical treatment options. Further studies are warranted to understand why lymphadenopathy and PLE develop despite ERT in some patients. Alternative treatment modalities should be investigated for treatment of poorly accessible organs including central nervous system, lungs and lymph nodes which are involved in GD.

\section{Author contribution}

The authors confirm contribution to the paper as follows: Conception and design, data collection, interpretation of data, draft manuscript preparation: MAG, EG, HHG; preparation of tables and figures: EG, HHG; drafting and revising the article: $\mathrm{HD}, \mathrm{HO}$, INST, SG, AY; critical revision: AY. All authors reviewed the results and approved the final version of the manuscript.

\section{Conflict of interest}

The authors declare no conflict of interest. 


\section{REFERENCES}

1. Burrow TA, Cohen MB, Bokulic R, et al. Gaucher disease: progressive mesenteric and mediastinal lymphadenopathy despite enzyme therapy. J Pediatr 2007; 150: 202-206.

2. Lee BH, Kim DY, Kim GH, Cho KJ, Yoon HK, Yoo HW. Progressive mesenteric lymphadenopathy with protein-losing enteropathy; a devastating complication in Gaucher disease. Mol Genet Metab 2012; 105: 522-524.

3. Mhanni AA, Kozenko M, Hartley JN, Deneau M, El-Matary W, Rockman-Greenberg C. Successful therapy for protein-losing enteropathy caused by chronic neuronopathic Gaucher disease. Mol Genet Metab Rep 2016; 6: 13-15.

4. Abdelwahab M, SeifEldien HM. Mesenteric and mediastinal lymphadenopathy in Egyptian children with Gaucher disease types 1 and 3 treated with enzyme replacement therapy. J Pediatr Hematol Oncol 2015; 37: e316-e322.

5. Yagci B, Salor O, Yalcin B, Gürakan F, Güçer S, Büyükpamukçu M. Giant lymphadenopathy infiltrated by gaucher cells mimicking lymphoma. Pediatr Blood Cancer 2009; 52: 870-871.
6. Altarescu G, Hill S, Wiggs E, et al. The efficacy of enzyme replacement therapy in patients with chronic neuronopathic Gaucher's disease. J Pediatr 2001; 138: 539-547.

7. Burrow TA, Sun Y, Prada CE, et al. CNS, lung, and lymph node involvement in Gaucher disease type 3 after 11 years of therapy: clinical, histopathologic, and biochemical findings. Mol Genet Metab 2015; 114: 233-241.

8. Cox TM, Aerts JM, Belmatoug N, et al. Management of non-neuronopathic Gaucher disease with special reference to pregnancy, splenectomy, bisphosphonate therapy, use of biomarkers and bone disease monitoring. J Inherit Metab Dis 2008; 31: 319-336.

9. Kewcharoen J, Mekraksakit P, Limpruttidham N, et al. Budesonide for protein losing enteropathy in patients with fontan circulation: a systematic review and meta-analysis. World J Pediatr Congenit Heart Surg 2020; 11: 85-91. 